\title{
Jean Prévost le multiple, éd. Emmanuel Bluteau et François Ouellet
}

Paola Codazzi

\section{(2) OpenEdition}

Journals

\section{Edizione digitale}

URL: http://journals.openedition.org/studifrancesi/5419

DOI: 10.4000/studifrancesi.5419

ISSN: 2421-5856

\section{Editore}

Rosenberg \& Sellier

\section{Edizione cartacea}

Data di pubblicazione: 1 dicembre 2016

Paginazione: 561-562

ISSN: 0039-2944

\section{Notizia bibliografica digitale}

Paola Codazzi, « Jean Prévost le multiple, éd. Emmanuel Bluteau et François Ouellet », Studi Francesi [Online], 180 (LX | III) | 2016, online dal 01 janvier 2017, consultato il 18 septembre 2020. URL : http:// journals.openedition.org/studifrancesi/5419; DOI : https://doi.org/10.4000/studifrancesi.5419

Questo documento è stato generato automaticamente il 18 settembre 2020.

\section{(c) $(1) \odot \odot$}

Studi Francesi è distribuita con Licenza Creative Commons Attribuzione - Non commerciale - Non opere derivate 4.0 Internazionale. 


\title{
Jean Prévost le multiple, éd. Emmanuel Bluteau et François Ouellet
}

\author{
Paola Codazzi
}

\section{NOTIZIA}

Jean Prévost le multiple, sous la direction de Emmanuel BLUTEAU et François OUELLET, Rennes, Presses Universitaires de Rennes, 2015, «Interférences», 150 pp.

1 Il volume raccoglie gli Atti del convegno organizzato a Parigi il 6 dicembre del 2014, a settant'anni dalla morte di Jean Prévost. Come si intuisce dal titolo, che riprende un'espressione di Pierre Bost, un grande amico dello scrittore, l'obiettivo del libro è quello di fornire un'immagine il più possibile fedele di un autore eclettico, dai mille talenti, forse troppo ingiustamente dimenticato dalla critica. La bibliografia, inserita prima dell'indice conclusivo, è in questo senso emblematica; per quanto limitata a soli vent'anni di attività, la produzione di Jean Prévost si presenta vasta e articolata: dalla narrativa alla poesia, dai saggi critici alla traduzione, passando per un numero elevatissimo di articoli, pubblicati in riviste specialistiche o in giornali a grande tiratura. Come si legge nell'introduzione, a cura di Emmanuel BluTEAu e di François OUELLET, i nove articoli che compongono il volume riflettono proprio la varietà e la modernità dell'autore.

2 Il contributo di Hélène BATY-DeLALANDE (pp.17-30) si concentra sulla produzione romanzesca di Jean Prévost, con l'obiettivo di delineare uno dei tratti essenziali della sua scrittura, ovvero l'angoisse démocratique: per Prévost, è fondamentale che il libro raggiunga il lettore, che lo scuota e che lo influenzi. Anche il secondo articolo è dedicato al Prévost romanziere: François OUELLET (pp.31-45) si propone di valutare l'influenza di Stendhal nella costruzione dell'intrigue. Il terzo contributo, quello di Emmanuel BLUTEAU (pp. 47-64), analizza quattro testi di fiction, con lo scopo di mostrare 
come per Prévost sia impossibile separare la scrittura letteraria da quella giornalistica, due attività che gli appaiono come quelle di un Janus bifrons.

In un certo senso, l'articolo di Bluteau introduce i tre successivi, il cui centro di interesse è proprio la collaborazione di Prévost con alcuni giornali dell'epoca. Secondo Karine ABADIE (pp. 65-79), lo scrittore può considerarsi un critico cinematografico di qualità, poiché sulle pagine della rivista Nouvelles littéraires, ha saputo distinguersi per la sua acuità di giudizio. Bruno CURATolo (pp. 81-94) e Sophie RoBert (pp. 95-110) indagano i contributi di Prévost tra le pagine, rispettivamente, di Europe e del Navire d'Argent: se il primo analizza nello specifico gli articoli che trattano del rapporto foiraison, il secondo riassume le tappe essenziali dell'esperienza dello scrittore presso la rivista diretta da Adrienne Monnier.

Il contributo di Mireille BRANGÉ (pp. 111-121) si distingue da quelli già citati, e da quelli che seguono, per il fatto di non dedicarsi allo studio di un genere preciso. Nell'articolo, infatti, si cerca essenzialmente di ricostruire la storia dell'amicizia tra Jean Prévost e Ramon Fernandez, scrittore, critico e giornalista di simpatie comuniste.

Gli interventi di Jean-Luc MARTINET (pp. 123-134) e Alexis B ufFEt (pp. 135-148), che chiudono il volume, esplorano un'altra faccia della produzione di Prévost, quella dei saggi critici. Martinet tenta di dare una definizione precisa del termine essai e giunge a individuare in questo tipo di scrittura la stessa tensione, o force, che anima l'opera romanzesca. Buffet, invece, si concentra sull'analisi di Usonie, esquisse de la civilisation américaine (1939), uno degli ultimi testi dello scrittore, frutto delle riflessioni maturate durante un lungo soggiorno negli Stati Uniti (1936-37). È a ovest, in America, che Prévost ripone le proprie speranze di vedere realizzarsi un mondo migliore.

Purtroppo, lo scrittore non vivrà abbastanza per prendere coscienza dei limiti del proprio disegno utopico. Con l'aumentare della tensione in Europa, Prévost abbandonerà progressivamente la letteratura in favore di un impegno politico attivo. Le multiple, dunque, non solo per la sua straordinaria versatilità letteraria: Prévost ha saputo infatti essere allo stesso tempo scrittore di mestiere e uomo d'azione. Per mano dei tedeschi si interromperà prematuramente la vita di un resistente, di un letterato, di un intellettuale, sul cui pensiero e sulla cui opera, come i curatori del volume sottolineano a più riprese, è necessario tornare a riflettere. 